ISSN 1980-5098

\title{
USO DE ESPÉCIES DA ARBORIZAÇÃO URBANA NO BIOMONITORAMENTO DE POLUIÇÃO AMBIENTAL
}

\author{
USE OF SPECIES OF URBAN TREES IN ENVIRONMENTAL POLLUTION BIOMONITORING
}

\author{
Kaíque Mesquita Cardoso ${ }^{1}$ Alessandro de Paula ${ }^{2}$ José Soares dos Santos ${ }^{3}$ \\ Maria Lúcia Pires dos Santos ${ }^{4}$
}

\begin{abstract}
RESUMO
O biomonitoramento consiste na utilização de organismos vivos para apontar possíveis poluições ambientais. Objetivou-se neste estudo avaliar a influência da intensidade do tráfego urbano na disponibilidade de elementos e sólidos em suspensão, bem como identificar a potencialidade no acúmulo e retenção das substâncias em diferentes espécies arbóreas, utilizando suas folhas como biomonitores passivos e de acumulação. Como amostras utilizaram-se folhas de árvores coletadas em duas áreas no município de Vitória da Conquista - BA: com trânsito de veículos e atividades antrópicas moderadas; e em uma zona de maior tráfego e com atividades intensas. Foram determinados o material particulado (MP) depositado nas folhas pelo método gravimétrico e os elementos $\mathrm{Fe}, \mathrm{Cu}, \mathrm{Ni}, \mathrm{Co}$ e $\mathrm{Cd}$ por espectrometria de absorção atômica em forno de grafite (EAA FG). O Fe se distinguiu significativamente das demais variáveis, com concentrações superiores aos outros metais. Utilizaram-se análises estatísticas multivariadas para a interpretação dos dados. A análise de agrupamento hierárquico separou dois grupos de acordo com a espacialidade e a análise de componentes principais permitiu a explicação desta tendência amostral. Um grupo foi constituído por $\mathrm{MP}, \mathrm{Fe}$ e $\mathrm{Cd}$, pelo fato de serem originados do desgaste dos veículos e queima de combustíveis fósseis. As espécies da família Fabaceae apresentaram uma maior similaridade em relação ao elemento $\mathrm{Cu}$, isto, porque a atividade metabólica do $\mathrm{Cu}$ tem comportamento inversamente proporcional à concentração de nitrogênio. O trânsito de veículos influiu diretamente na disponibilidade de alguns elementos tóxicos e na concentração de partículas sólidas depositadas. Conclui-se que a espécie influi no acúmulo dos elementos químicos e apresentam diferentes potenciais de captação. A arborização pode ser utilizada como biomonitora passiva de acumulação.
\end{abstract}

Palavras-chave: Bauhinia variegata; Caesalpinia peltophoroides; Terminalia catappa; EAA FG.

\section{ABSTRACT}

Biomonitoring is the use of living organisms to identify possible environmental pollution. The objective of this study was to evaluate the influence of intensity of urban traffic in the availability of elements and suspended solids as well as identify the potential in the accumulation and retention of substances in different tree species using its leaves as passive biomonitors and for accumulation. As samples we used tree leaves collected from two areas in the municipality of Vitoria da Conquista (BA state), with transit vehicles and

1 Engenheiro Florestal e Professor do Ensino Básico, Técnico e Tecnológico do Instituto Federal do Norte de Minas Gerais. Fazenda do Meio Pé de Serra, S/N, BR 367, km 278, CEP 39600-000, Araçuaí (MG), Brasil. kaique. cardoso@ifnmg.edu.br

2 Engenheiro Florestal, Dr. Professor do Departamento de Engenharia Agrícola e Solos, Universidade Estadual do Sudoeste da Bahia, Estrada do Bem Querer, km 4, CEP 45031-900, Vitória da Conquista (BA), Brasil. apaula@uesb.edu.br

3 Químico Analítico e Ambiental, Dr., Professor do Departamento de Ciências Naturais, Universidade Estadual do Sudoeste da Bahia, Estrada do Bem Querer, km 4, CEP 45031-900, Vitória da Conquista (BA), Brasil. zesoares@uesb.edu.br

4 Química, $\mathrm{Dr}^{\mathrm{a}}$., Professora do Departamento de Ciências Naturais, Universidade Estadual do Sudoeste da Bahia, Estrada do Bem Querer, km 4, CEP 45031-900, Vitória da Conquista (BA), Brasil. luciapires@uesb.edu.br

Recebido para publicação em 28/12/2014 e aceito em 01/10/2015. 
moderate human activities, and in a zone of increased traffic and intense activities. We determined the particulate matter (MP) deposited in the leaves by the gravimetric method and the elements $\mathrm{Fe}, \mathrm{Cu}, \mathrm{Ni}$, $\mathrm{Co}$ and $\mathrm{Cd}$ by atomic absorption spectrometry with graphite furnace (GF AAS). Fe was significantly distinguished from other variables, with higher concentrations than other metals. The data was interpreted by multivariate analysis. Cluster analysis separated two groups, one consisting of MP, $\mathrm{Fe}$ and $\mathrm{Cd}$, because they are originated from the wear and tear of vehicles and burning fossil fuels. The species of the family Fabaceae showed greater similarity to $\mathrm{Cu}$ element, namely, because the $\mathrm{Cu}$ metabolic activity is inversely proportional to the concentration of nitrogen behavior. The vehicle traffic directly influenced the availability of some toxic elements and the concentration of the deposited solid particles. It is concluded that the species influences the accumulation of chemical elements and have different catchment potentials. The trees can be utilized as biomonitors which are passive of accumulation.

Keywords: Bauhinia variegata; Caesalpinia peltophoroides; Terminalia catappa; GF AAS.

\section{INTRODUÇÃO}

Os elementos tóxicos sempre foram encontrados e ocorreram de forma natural no meio ambiente (ESTEVES, 1988), contudo, algumas atividades sociais têm contribuído de maneira efetiva para os aumentos significativos desses contaminantes em distintos ecossistemas, permitindo que estes se tornem disponíveis em recursos hídricos, solos e atmosfera. Tal problemática socioambiental é um fato que deve ser investigado, visto que, ao depender das concentrações de metais pesados em contato com o organismo, proporciona diversos riscos à saúde devido à exposição (SATARUG et al., 2010).

Qualquer substância com potencialidade de contaminação e que possa causar danos em seres vivos e materiais é considerada poluente. Esta pode advir tanto de fenômenos naturais quanto das atividades humanas. Esses poluentes do ar são emitidos e podem ser encontrados na forma gasosa ou como material particulado e metais (KLUMPP et al., 2001; BRASIL, 2011).

O material particulado, os gases e os metais tóxicos, são originados principalmente por resíduos industriais e veículos automotores. Veículos movidos a diesel apresentam a maior concentração média de emissão de partículas, seguidas pela gasolina (três vezes menos) e etanol (sete vezes menos). A constituição do combustível explica esses resultados, pois, além de ser formado por hidrocarbonetos pesados e altos teores de metais como o enxofre, o óleo diesel ainda necessita de alta quantidade de ar aquecido para promover a ignição. Além do enxofre permitir maior formação de partículas, os três combustíveis apresentam como composição elementar os metais ferro $(\mathrm{Fe})$, cobre $(\mathrm{Cu})$ e chumbo $(\mathrm{Pb})$. Sendo que, ao depender da particularidade do combustível e do tipo de partícula (fina ou grossa), também são encontrados os metais alumínio $(\mathrm{Al})$, silício $(\mathrm{Si})$, potássio $(\mathrm{K})$, cálcio $(\mathrm{Ca})$, crômio $(\mathrm{Cr})$, manganês $(\mathrm{Mn})$, níquel $(\mathrm{Ni})$ e zinco (Zn). Para atender às exigências do mercado consumidor, a gasolina, antes de ser distribuída é enriquecida com aditivos (corantes, agentes antidetonantes, antioxidantes, inibidores de corrosão e elevadores de volatilidade). Estes compostos contêm elementos-traço como $\mathrm{Fe}, \mathrm{Cr}, \mathrm{Cu}$ e $\mathrm{Pb}$, que advêm, em sua maior parte, dos óleos antes do refino (SILVA, 2007).

Dessa forma, pode-se perceber o alto grau de comprometimento do ambiente urbano devido à emissão e acúmulo dessas partículas. Tanto a identificação desses poluentes, como a determinação de suas concentrações, é fundamental para a elaboração de políticas públicas e tomadas de decisão quanto a seus mecanismos de emissão.

Neste contexto, o uso de bioindicadores de poluição ambiental é imperioso, pois permitem, segundo Klumpp et al. (2001), detectar os efeitos dos poluentes sobre os organismos e inferir sobre a qualidade do ar. Sendo assim, o uso da arborização urbana como bioindicadora é o ideal, já que são componentes filtrantes dessas partículas e gases e estão expostas diuturnamente às fontes emissoras.

As plantas podem ser usadas como indicadoras de reação ou como indicadoras de acumulação. As indicadoras de reação são aquelas que em contato com a poluição respondem por meio de deformações na anatomia, apresentando danos visíveis em suas estruturas. As indicadoras de acumulação são resistentes à poluição, acumulam substâncias tóxicas nos tecidos foliares e permitem a deteç̧ão do poluente por meio de análises químicas. Quanto à obtenção da espécie para fins do biomonitoramento, podem ser ativa quando se expõe uma espécie por um determinado período, e passiva, quando as análises são realizadas em espécies 
já existentes no meio (MARTINS, 2009).

Sendo assim, o objetivo deste estudo foi avaliar a influência da intensidade do tráfego urbano na disponibilidade de elementos e sólidos em suspensão, bem como identificar a potencialidade no acúmulo e retenção das substâncias em diferentes espécies arbóreas, utilizando suas folhas como biomonitores passivos e de acumulação.

\section{MATERIAL E MÉTODOS}

\section{Áreas de estudo}

O estudo foi realizado em duas áreas localizadas no município de Vitória da Conquista - BA. Uma situa-se no campus da Universidade Estadual do Sudoeste da Bahia (UESB). Esta área foi selecionada, pois apresenta trânsito de veículos e com atividades antrópicas moderadas. Neste trabalho é representada pela letra "C". A outra área selecionada foi a Avenida Olívia Flores no Bairro Candeias. Esta é uma zona de maior tráfego urbano e com atividades intensas, sendo representada pela letra "U" (Figura 1).

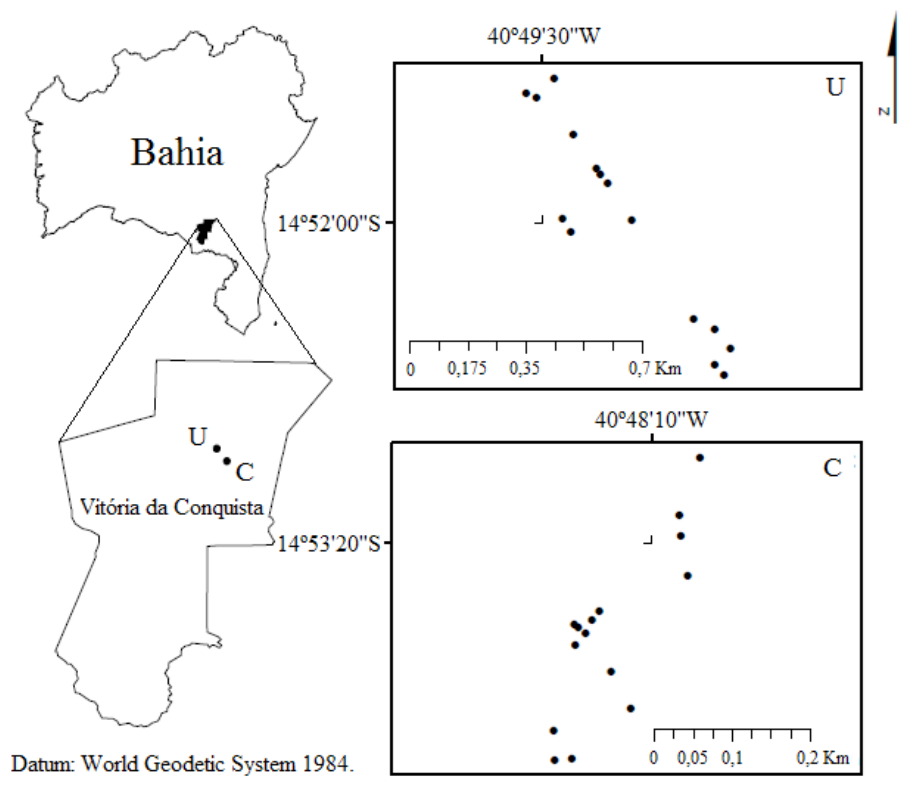

FIGRURA 1: Localização das árvores amostradas nas áreas em estudo: U (avenida) e C (campus). FIGURE 1: Location of the trees in the areas of the study: $U$ (avenue) and $C$ (campus).

Segundo a classificação climática de Köppen, a região estudada é do tipo Aw quente com estação seca bem acentuada e precipitações anuais em média de $700 \mathrm{~mm}$. O clima é do tipo Tropical de Altitude, cuja temperatura média no mês mais quente é de $22,1^{\circ} \mathrm{C}$, no mês mais frio é de $15,8^{\circ} \mathrm{C}$, e com média anual de $20^{\circ} \mathrm{C}$. A vegetação pertence à tipologia de Floresta Estacional variando de Semidecidual à Decidual (SOARES FILHO, 2000).

A cidade de Vitória da Conquista possui uma população de 306.866 pessoas, com uma densidade demográfica de 91,41 habitantes $/ \mathrm{km}^{2}$ e com $90 \%$ da população residente no meio urbano (IBGE, 2013). A frota na cidade para o mês de dezembro de 2013 somou um total de 104.954 veículos, sendo esta a terceira maior frota de veículos do estado da Bahia (BRASIL, 2013).

\section{Amostragem}

Foram coletadas folhas de três espécies florestais, sendo: 1 - Amendoeira (Terminalia catappa L., Família Combretaceae); 2 - Sibipiruna (Caesalpinia peltophoroides Benth., Família Fabaceae) e 3 - Patade-vaca (Bauhinia variegata L., Família Fabaceae). Cada amostra foi composta por três ramos de cinco 
árvores. A coleta foi realizada em dezembro de 2013, com o propósito de avaliar a influência espacial da poluição na qualidade ambiental, realizou-se a coleta apenas neste período. Este mês se apresentou com chuvas regulares em toda a região.

A seleção das folhas foi padronizada para compor o universo amostral. Com o auxílio de um podão, foram coletados ramos entre 2,0 e 3,0 metros de altura na parte exterior da copa.

\section{Preparo das amostras}

\section{Determinação do Material Particulado}

Logo após coletadas, as folhas foram acondicionadas em sacos plásticos estéreis e levadas ao laboratório. A determinação do material particulado foi realizada por meio do método gravimétrico (BRAUN; APPEL; SCMAL, 2003; INOUE; REISSMANN, 1991; MENEZES; CATALUÑA, 2008). Prepararam-se primeiramente filtros quantitativos. Estes foram previamente secos em estufa até peso constante. Foram cortadas e pesadas 3,0 $\pm 0,001 \mathrm{~g}$ das folhas homogeneizadas. Em seguida, estas foram lavadas sobre os filtros e enviados novamente para estufa e secas até peso constante. Por diferença entre as massas inicial e final, obteve-se a quantidade de material particulado depositados nas folhas.

\section{Digestão das Amostras}

A segunda etapa de preparação das amostras consistiu inicialmente de sua secagem na estufa em bandejas de alumínio à temperatura de $60^{\circ} \mathrm{C}$ por 48 horas. Assim, com as amostras de folhas secas, estas foram trituradas com o propósito de facilitar o processo de extração. Neste processo, as amostras foram submetidas a uma digestão ácida assistida por micro-ondas, em triplicata (CARODE et al., 2008; VACCARO et al., 2004; CARNEIRO et al., 2006; MELO; SILVA, 2008).

Pesou-se $1 \mathrm{~g}$ da amostra em balança analítica, adicionada a esta $6 \mathrm{~mL}$ de $\mathrm{HNO}_{3} 65 \%$ em frascos fechados de Teflon. A amostra então foi levada ao micro-ondas pressurizado em uma proveta analítica (modelo DGT 100 plus), para a execução do programa de aquecimento apresentado na Tabela 1. O método de digestão foi baseado no manual de preparação de amostra do digestor por micro-ondas.

TABELA 1: Programa de aquecimento utilizado para digestão assistida em forno de micro-ondas.

TABLE 1: Heating program used for assisted digestion in a microwave oven.

\begin{tabular}{ccc}
\hline Etapa & Tempo $(\min )$ & Potência $(\mathrm{W})$ \\
\hline 1 & 7 & 330 \\
2 & 2 & 800 \\
3 & 5 & 500 \\
4 & 10 & 0 \\
\hline
\end{tabular}

Posteriormente, as amostras foram avolumadas diretamente em balões de $25 \mathrm{~mL}$. Ao completar o seu volume com água ultrapura obtida pelo sistema Milli-Q, foram transferidas para fracos de polietileno.

Com o intuito de evidenciar a interferência dos reagentes, realizou-se um ensaio em branco contento a solução extratora e a água ultrapura, seguindo o mesmo fluxograma de preparo da amostra.

\section{Procedimento Analítico}

\section{Espectrometria de absorção atômica em forno de grafite (GF AAS)}

As determinações de Cd, Ni, Co e Fe foram realizadas por GF AAS, Perkin-Elmer Precisely modelo AAnalyst 600, em triplicata com protocolo de análise sugerido pelo fabricante na Tabela 2. Para a preservação da amostra em temperaturas de pirólise maiores e eliminação de interferentes da matriz,

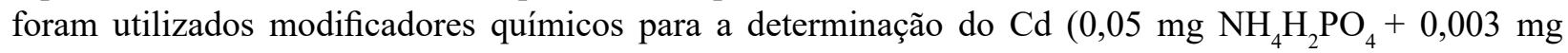


TABELA 2: Programação do aquecimento do GF AAS, sendo: $\operatorname{Tr}=$ tempo de rampa em segundos, $\mathrm{Te}=$ tempo de espera em segundos e $\mathrm{Fi}=$ fluxo interno.

TABLE 2: Programming warming GF AAS, as follows: $\mathrm{Tr}=$ ramp time in seconds, $\mathrm{Te}=$ timeout in seconds and $\mathrm{Fi}=$ internal flow.

\begin{tabular}{|c|c|c|c|c|c|c|c|c|}
\hline \multirow{2}{*}{ Etapas } & \multicolumn{5}{|c|}{ Temperatura $\left({ }^{\circ} \mathrm{C}\right)$} & \multirow{2}{*}{$\operatorname{Tr}$} & \multirow{2}{*}{$\mathrm{Te}$} & \multirow{2}{*}{$\mathrm{Fi}$} \\
\hline & $\mathrm{Cd}$ & $\mathrm{Fe}$ & $\mathrm{Ni}$ & Co & $\mathrm{Cu}$ & & & \\
\hline 1.Secagem & & & 110 & & & 1 & 30 & 250 \\
\hline 2.Secagem & & & 130 & & & 15 & 30 & 250 \\
\hline 3.Pirólise & 500 & 1440 & 1110 & 1400 & 1200 & 10 & 20 & 250 \\
\hline 4.Atomização & 1500 & 2100 & 2300 & 2400 & 2000 & 0 & 5 & 0 \\
\hline 5.Limpeza & 2450 & 2450 & 2450 & 2450 & 2450 & 1 & 3 & 250 \\
\hline
\end{tabular}

$\left.\mathrm{Mg}\left(\mathrm{NO}_{3}\right)_{2}\right)$.

No equipamento foram introduzidos poucos microlitros no forno pela pipeta automática. Em seguida, a amostra foi submetida a uma série de etapas programadas (secagem, pirólise, atomização e limpeza). Na etapa de secagem, com temperaturas relativamente baixas, o solvente da amostra evaporou e logo após com temperaturas mais elevadas ocorreu a calcinação ou a conversão da matéria orgânica em $\mathrm{H}_{2} \mathrm{O}$ e $\mathrm{CO}_{2}$. Em seguida ocorreu a etapa de atomização, sendo a amostra vaporizada e atomizada. Posteriormente, ocorreu um rápido aumento da temperatura, cerca de $2.500^{\circ} \mathrm{C}$. A partir daí a atomização ocorreu em poucos segundos. Sendo assim, a técnica consistiu em gerar uma nuvem densa de átomos, que em condições controladas, permitiu a absorção das partículas atomizadas, medida em uma região acima da superfície de aquecimento. A última etapa foi a da limpeza, com temperaturas muito altas utilizadas para evitar o efeito memória do analito (SKOOG et al., 2007).

A calibração realizada foi do tipo externa. Os limites de detecção e quantificação foram calculados segundo as equações 1 e 2, respectivamente (RIBANI et al., 2004). Em que, "s" representa o desvio padrão da resposta de cinco leituras do branco, e "S" representa a inclinação da equação da reta.

$$
\begin{aligned}
& \mathrm{LD}=3,3 \mathrm{~s} / \mathrm{S}(1) \\
& \mathrm{LQ}=10 \mathrm{~s} / \mathrm{S}(2)
\end{aligned}
$$

\section{Análises Estatísticas}

Devido à existência de fatores como a morfologia das folhas e localidade das amostras, que poderiam afetar a quantidade de elementos químicos depositados, as análises de agrupamento hierárquico (AAH) e de componentes principais (ACP) foram aplicadas nos resultados analíticos com o propósito de obter variáveis capazes de caracterizar a originalidade das amostras sob a influência das atividades citadinas. Todos os cálculos foram realizados com o auxílio dos programas SPSS 16.0.

Para a interpretação dos dados, foi gerada uma matriz de dados $6 \times 6$, na qual as linhas corresponderam às amostras de folhas (casos) e as colunas as variáveis $\mathrm{MP}, \mathrm{Fe}, \mathrm{Ni}, \mathrm{Cu}, \mathrm{Co}$ e $\mathrm{Cd}$. Como as variáveis não possuíam a mesma escala, foi necessário escalonar os dados por meio do método Z-Score. A medida de similaridade usada foi a distância euclidiana e o método hierárquico aglomerativo utilizado foi o método de ligação de Ward.

\section{Análise de Agrupamento Hierárquico}

Portando-se das concentrações médias de cada ponto amostral, foi utilizada a análise hierárquica de cluster. Este é um método numérico multivariado, que possui como objetivo de propor uma estrutura classificatória ou de reconhecimento da existência de grupos, baseado na similaridade das variáveis e a 
diferença entre os membros de classes distintas (REGASSI, 2001).

Para a representação gráfica dos resultados optou-se pela utilização de um dendrograma. A similaridade entre as amostras foi medida por distância euclidiana.

\section{Análise de Componentes Principais}

Nesta análise o princípio fundamental foi baseado na redução da dimensão do conjunto dos resultados analíticos, em que se preservou a maior quantidade de informação (variância). Assim, para a análise de componentes principais, foram avaliadas as principais influências das variáveis no comportamento de grupos.

Na ACP considerou-se um método fatorial, pois a redução do número de variáveis foi feita pelo estabelecimento de novas variáveis ortogonais entre si, denominadas de componentes principais $(\mathrm{CP})$ e foram obtidas pela combinação linear das variáveis iniciais, por meio de fatores.

Os componentes principais foram organizados em ordem decrescente de importância e os gráficos foram obtidos através da análise das amostras em um sistema cartesiano no qual os seus eixos são as CP.

\section{Análise de Correlação}

Para a observação das correlações e seus respectivos graus de importância, aplicou-se a partir da matriz de dados das concentrações médias das variáveis uma análise de correlação com base no coeficiente

TABELA 3: Médias de concentração das variáveis da leitura em triplicata das amostras de folha seguidas pelo desvio padrão, sendo: $\mathrm{C} 1$ = indivíduo de Terminalia catappa situado no campus, $\mathrm{C} 2=$ indivíduo de Caesalpinia peltophoroides situado no campus, C3 = indivíduo de Bauhinia variegata situado no campus, U1 = indivíduo de Terminalia catappa situado na avenida, U2 = indivíduo de Caesalpinia peltophoroides situado na avenida, U3 = indivíduo de Bauhinia variegata situado na avenida; $\mathrm{LD}=$ limite de detecção.

TABLE 3: Mean concentration of variables in triplicate of swatches followed by standard deviation, where: $\mathrm{C} 1=$ Terminalia catappa located on campus, $\mathrm{C} 2=$ Caesalpinia peltophoroides located on campus, $\mathrm{C} 3$ = Bauhinia variegate located on campus, U1 = Terminalia catappa located in the avenue, $\mathrm{U} 2=$ Caesalpinia peltophoroides located in the avenue, $\mathrm{U} 3=$ Bauhinia variegata located in the avenue; $\mathrm{LD}=$ detection limit.

\begin{tabular}{cccc}
\hline Amostra & $\mathrm{MP}\left(\mathrm{g} \mathrm{Kg}^{-1}\right)$ & $\mathrm{Cd}\left(\mathrm{mg} \mathrm{Kg}^{-1}\right)$ & $\mathrm{Co}\left(\mathrm{mg} \mathrm{Kg}^{-1}\right)$ \\
\hline $\mathrm{C} 1$ & $2,001 \pm 0,001$ & $0,018 \pm 0,000$ & $0,179 \pm 0,002$ \\
$\mathrm{C} 2$ & $3,009 \pm 0,005$ & $<\mathrm{LD}$ & $0,097 \pm 0,006$ \\
$\mathrm{C} 3$ & $2,271 \pm 0,001$ & $<\mathrm{LD}$ & $0,138 \pm 0,008$ \\
$\mathrm{U} 1$ & $3,673 \pm 0,007$ & $0,053 \pm 0,005$ & $0,198 \pm 0,013$ \\
$\mathrm{U} 2$ & $3,678 \pm 0,006$ & $0,067 \pm 0,007$ & $0,113 \pm 0,007$ \\
$\mathrm{U} 3$ & $4,011 \pm 0,011$ & $0,048 \pm 0,004$ & $0,230 \pm 0,012$ \\
\hline Amostra & $\mathrm{Cu}\left(\mathrm{mg} \mathrm{Kg}^{-1}\right)$ & $\mathrm{Fe}\left(\mathrm{mg} \mathrm{Kg}^{-1}\right)$ & $\mathrm{Ni}\left(\mathrm{mg} \mathrm{Kg}^{-1}\right)$ \\
\hline C1 & $0,789 \pm 0,034$ & $803,933 \pm 12,887$ & $1,680 \pm 0,064$ \\
C2 & $0,344 \pm 0,011$ & $1100,100 \pm 3,536$ & $1,426 \pm 0,083$ \\
C3 & $<\mathrm{LD}$ & $540,100 \pm 3,122$ & $0,501 \pm 0,030$ \\
$\mathrm{U} 1$ & $0,788 \pm 0,057$ & $1498,933 \pm 5,575$ & $1,872 \pm 0,070$ \\
$\mathrm{U} 2$ & $0,279 \pm 0,006$ & $1645,600 \pm 9,014$ & $1,260 \pm 0,046$ \\
$\mathrm{U} 3$ & $<\mathrm{LD}$ & $2069,267 \pm 2,021$ & $1,786 \pm 0,088$ \\
\hline
\end{tabular}


de correlação de Pearson.

\section{RESULTADOS E DISCUSSÃO}

As médias das determinações do material particulado e elementos químicos demonstraram que tanto o local quanto a espécie influenciaram nos resultados (Tabela 3). O comportamento das variáveis foi dinâmico, expondo a influência e especialidade dos elementos químicos.

Alguns elementos-traço não foram quantificados, pois permaneceram abaixo do limite de detecção (LD) do método para a técnica GF AAS (Tabela 4). O Cd em algumas amostras oriundas do campus e o Cu para Terminalia catappa (Tabela 3 ).

O Fe se distinguiu significativamente das demais variáveis, com concentrações muito superiores aos outros metais (Tabela 3). Isso pode ser explicado devido à presença deste elemento em todos os tipos de combustíveis e partículas de fuligem (SILVA, 2007). O Fe também está relacionado com a poeira e ressuspensão do material particulado, além de estar presente na composição química da terra e ser gerado no desgaste de peças metálicas dos veículos (MOREIRA, 2010).

Verificou-se que este elemento apresentou teores elevados quando comparados com estudos semelhantes realizados por outros autores. Para Silva (2011), que estudou contaminantes atmosféricos em avenidas principais da cidade de Patos - PB, as concentrações mais elevadas de Fe variaram em torno de 444,00 mg Kg-1. Já Moreira (2010) quantificou teores médios de 199,72 $\mathrm{mg} \mathrm{Kg}^{-1}$ do elemento em folhas de árvores localizadas no parque Ibirapuera da cidade de São Paulo - SP. A área de estudo e a metodologia aplicada, respectivamente, explicam os altos teores neste estudo. A cidade de Patos, de acordo com o autor, possui uma frota de 24.123 veículos, cerca de quatro vezes menor do que Vitória da Conquista BA (SILVA, 2011). Moreira (2010), que objetivou estudar a absorção de elementos pela folha, lavou-as com o propósito de evitar a contaminação de superfície e assim reduzindo provavelmente os teores de acumulação. Concentrações superiores foram determinadas por Martins (2009), que, ao estudar a influência de corredores de tráfego veicular em parques da cidade de São Paulo, por meio do acúmulo de metais-traços em cascas de árvores, obteve concentrações superiores a $5.000 \mathrm{mg} \mathrm{Kg}^{-1} \mathrm{em}$ locais de influência citadina e inferiores a $106 \mathrm{mg} \mathrm{Kg}^{-1} \mathrm{em}$ área distantes de corredores de tráfego veicular.

Para os elementos $\mathrm{Cu}, \mathrm{Cd}$ e $\mathrm{Co}$, foram apuradas concentrações inferiores quando comparados na literatura. Moreira (2010) obteve teores na média de 9,38 $\mathrm{mg} \mathrm{Kg}^{-1}$ de $\mathrm{Cu}$ e $0,23 \mathrm{mg} \mathrm{Kg}^{-1}$ de Cd em folhas de árvores. Para Martins (2009), as variações da concentração de $\mathrm{Cu}$ mostraram influência da intensidade do tráfego veicular na acumulação do elemento em cascas de árvores, sendo inferior a $20 \mathrm{mg} \mathrm{Kg}^{-1}$ em área

TABELA 4: Parâmetros analíticos para técnica de GF AAS: Coeficiente de determinação $\left(\mathrm{R}^{2}\right)$, desvio padrão da resposta analítica do branco (DP branco), limite de detecção (LD) e limite de quantificação (LQ) do método.

TABLE 4: Analytical parameters for GF AAS technique: Determination coefficient $\left(\mathrm{R}^{2}\right)$, standard deviation of analytical response of white (DP branco), limit of detection (LD) and limit of quantification (LQ) of the method.

Elemento $\quad \mathrm{R}^{2} \quad$ Inclinação da curva $\quad \mathrm{DP}$ Branco $(\mathrm{n}=5) \quad \mathrm{LD}\left(\mathrm{mg} \mathrm{Kg}{ }^{-1}\right) \quad \mathrm{LQ}\left(\mathrm{mg} \mathrm{Kg}^{-1}\right)$

\begin{tabular}{llllll}
\hline $\mathrm{Cd}$ & 0,9957 & 0,0062 & 0,0024 & 0,0013 & 0,0039 \\
$\mathrm{Co}$ & 0,9993 & 0,0039 & 0,0001 & 0,0001 & 0,0004 \\
$\mathrm{Cu}$ & 0,9907 & 0,0028 & 0,0004 & 0,0004 & 0,0012 \\
$\mathrm{Fe}$ & 0,9993 & 0,0022 & 0,0015 & 0,0022 & 0,0067 \\
$\mathrm{Ni}$ & 0,9964 & 0,0040 & 0,0039 & 0,0032 & 0,0096 \\
\hline
\end{tabular}


controlada, e superior a $90 \mathrm{mg} \mathrm{Kg}^{-1} \mathrm{em}$ parques urbanos. Já o Co apresentou 0,2 $\mathrm{mg} \mathrm{Kg}^{-1} \mathrm{em}$ área controlada e 3,9 $\mathrm{mg} \mathrm{Kg}^{-1} \mathrm{em}$ amostras oriundas de local próximo aos corredores de tráfego veicular. De acordo com Moreira (2010), a provável fonte de Cu na atmosfera de emissão veicular está relacionada ao desgaste de peças, em relação ao $\mathrm{Cd}$, a principal fonte é devido ao atrito dos pneus. Aponta-se que os teores de ordem inferior neste estudo são devidos à pequena frota de veículos da cidade de Vitória da Conquista, quando comparados aos estudos realizados na cidade de São Paulo - SP, que possuía registro de 6.673.882 veículos, 63 vezes maior que a área deste estudo (MOREIRA, 2010).

Os teores de Ni obtidos neste estudo foram inferiores aos encontrados por Martins (2009), que relatou 5,0 $\mathrm{mg} \mathrm{Kg}^{-1}$ do elemento presente em cascas de árvores oriundas de uma área controlada e 15,1 mg $\mathrm{Kg}^{-1}$ no meio urbano. Por permanecer em geral por um período mais longo na planta, as cascas das árvores tendem a acumular maiores concentrações de poluentes atmosféricos.

Especialmente em relação ao material particulado, foi possível observar ainda no momento das análises a distinção entre as espécies do campus e as da avenida pelo acúmulo de material particulado nos filtros (Figura 2). Verificou-se que houve uma maior acumulação de material particulado nas folhas de árvores localizadas na avenida, com uma proporção que variou entre 1,2 a 1,8 maior para com o campus, apontando assim o efeito poluidor do tráfego de veículos. Inoue e Reissmann (1991), ao verificar o efeito da descarga de veículos automotores na deposição de particulados em folhas de Ligustrum lucidum, constataram que a amostra coletada em local de intenso movimento de veículos apresentou um pouco mais que o dobro de conteúdo de matéria sólida em relação à amostra oriunda de um bosque da periferia da cidade $\left(445 \mathrm{mg} / \mathrm{m}^{2}\right)$. De acordo com Carneiro (2004), o material particulado ou partículas inaláveis são produzidos nos processos de combustão veicular.

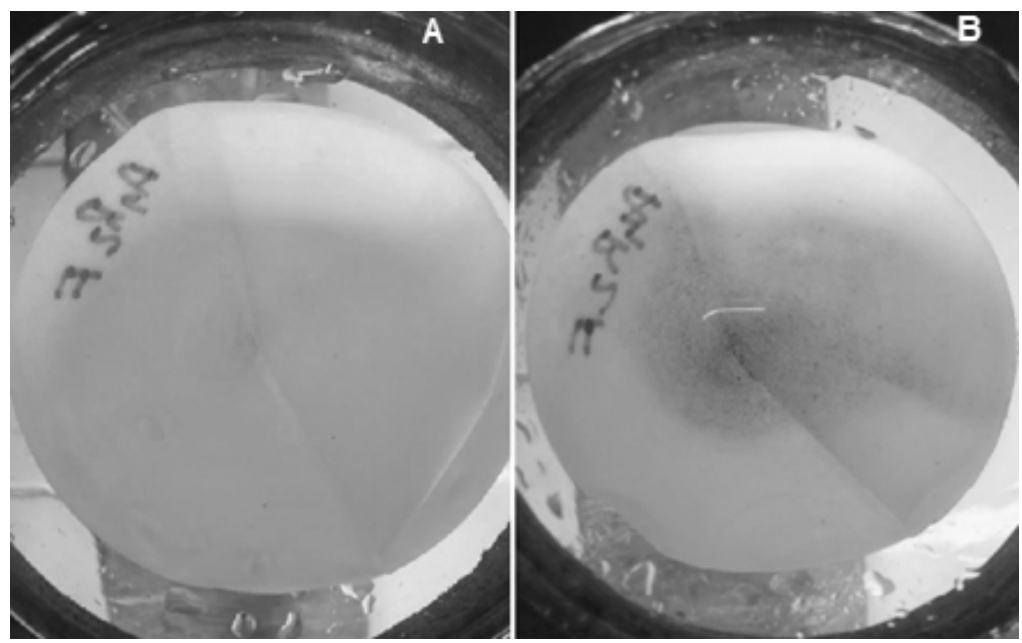

FIGURA 2: Material particulado retido no papel-filtro após lavagem de uma mesma espécie em locais distintos: campus (A) e avenida (B).

FIGURE 2: Particulate matter trapped in the filter paper after washing of the same species in different locations: campus (A) and avenue (B).

\section{Análise de Agrupamento Hierárquico}

$\mathrm{Na}$ avaliação do dendrograma foi possível observar a formação de dois grupos principais (Figura 3A). O primeiro grupo foi formado por amostras originadas do campus. O grupo II, formado somente por amostras da avenida, apresentou uma maior similaridade. Tal fato pode ser explicado em função da localidade das amostras, sendo as da avenida, influenciadas pela maior exposição das árvores aos contaminantes na zona de maior tráfego.

Em relação à análise de agrupamento por concentração de variáveis (Figura 3B), também se pôde observar a formação de dois grupos. Sendo o primeiro constituído pelas variáveis MP, Fe e Cd. A formação deste grupo pode ser explicada em função da fonte do material particulado depositado. Esse material 


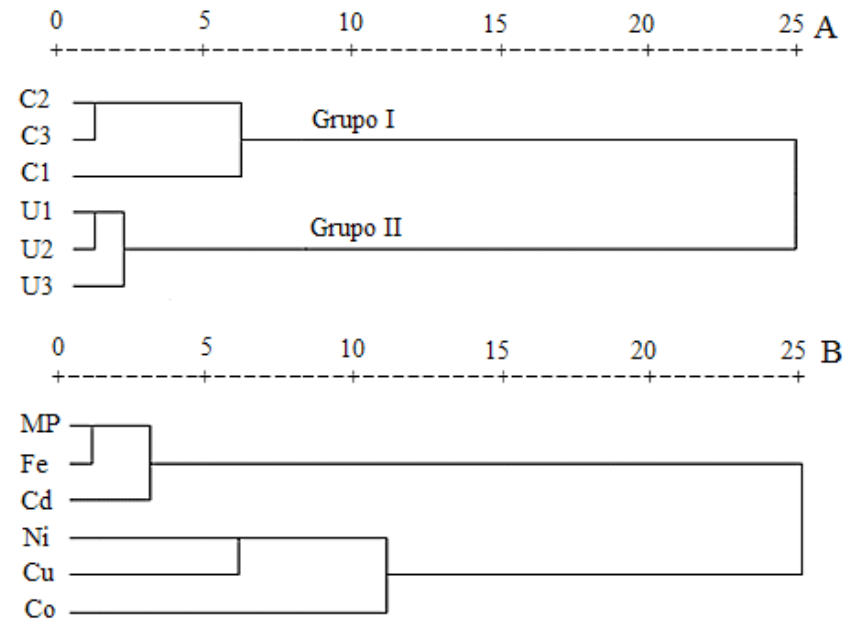

FIGURA 3: Dendrogramas de similaridade (A) por amostras coletadas no campus da Universidade Estadual do Sudoeste da Bahia e na avenida Olívia Flores e (B) por concentração de elementos químicos. C1 = indivíduo de Terminalia catappa situado no campus, $\mathrm{C} 2$ = indivíduo de Caesalpinia peltophoroides situado no campus, C3 = indivíduo de Bauhinia variegata situado no campus, U1 = indivíduo de Terminalia catappa situado na avenida, U2 = indivíduo de Caesalpinia peltophoroides situado na avenida, U3 = indivíduo de Bauhinia variegata situado na avenida

FIGURE 3: Dendrograms of similarity (A) for samples collected on the campus of the State University of Southwest Bahia and Avenue Olivia Flores and (B) by concentration of chemical elements. C1 = indivíduo de Terminalia catappa situado no campus, $\mathrm{C} 2$ = indivíduo de Caesalpinia peltophoroides situado no campus, C3 = indivíduo de Bauhinia variegata situado no campus, U1 = indivíduo de Terminalia catappa situado na avenida, U2 = indivíduo de Caesalpinia peltophoroides situado na avenida, U3 = indivíduo de Bauhinia variegata situado na avenida

depositado foi gerado principalmente pelo desgaste de peças dos automóveis, as quais contêm ferro na sua composição, aglutinado ao cádmio, originado provavelmente dos processos de galvanoplastia, sendo a cromagem um artifício comum em peças de veículos (MOORE; RAMAMOORTHY, 1984), bem como pela queima de combustíveis fósseis (MATTHEWS, 1984; DUARTE; PASQUAL, 2000). Já o grupo II formouse a partir das demais variáveis com subgrupos caracterizados principalmente pela origem e especificidade química da natureza dos elementos.

\section{Análise de Componentes Principais}

Foram selecionados dois componentes $(\mathrm{CP} 1$ x CP2) derivados por rotação varimax, o segundo componente concentrou $27,01 \%$ da variância total e o primeiro componente $54,24 \%$, totalizando $81,25 \%$ de variância total (Figura 4).

No gráfico de escores (Figura 4A) pode ser observada a distribuição das amostras, as quais formaram dois grupos, novamente separando amostras do campus e da avenida, mostrando assim uma influência direta no comportamento dos dados referente à espacialidade. Ao longo do eixo da CP1 é possível observar que as amostras do campus apresentaram valores negativos, enquanto que nas amostras da avenida foram obtidos valores positivos. Sendo assim, o gráfico de escore corrobora com a análise de agrupamento do dendrograma.

O gráfico de loadings, representado na Figura 4B, permite a caracterização de tendências entre as variáveis e quanto elas influenciaram o sistema e comportamento das amostras. Ao longo do eixo da $\mathrm{CP} 1$, pode-se observar que a maioria dos elementos proporcionaram e explicaram a distinção referente aos dois grupos, todos com valores positivos. $\mathrm{O} F$, $\mathrm{Cd}$ e MP contribuíram para uma maior diferenciação dos agrupamentos, juntamente com o Co e Ni. Contudo, é notório que o elemento $\mathrm{Cu}$ distanciou-se com um 

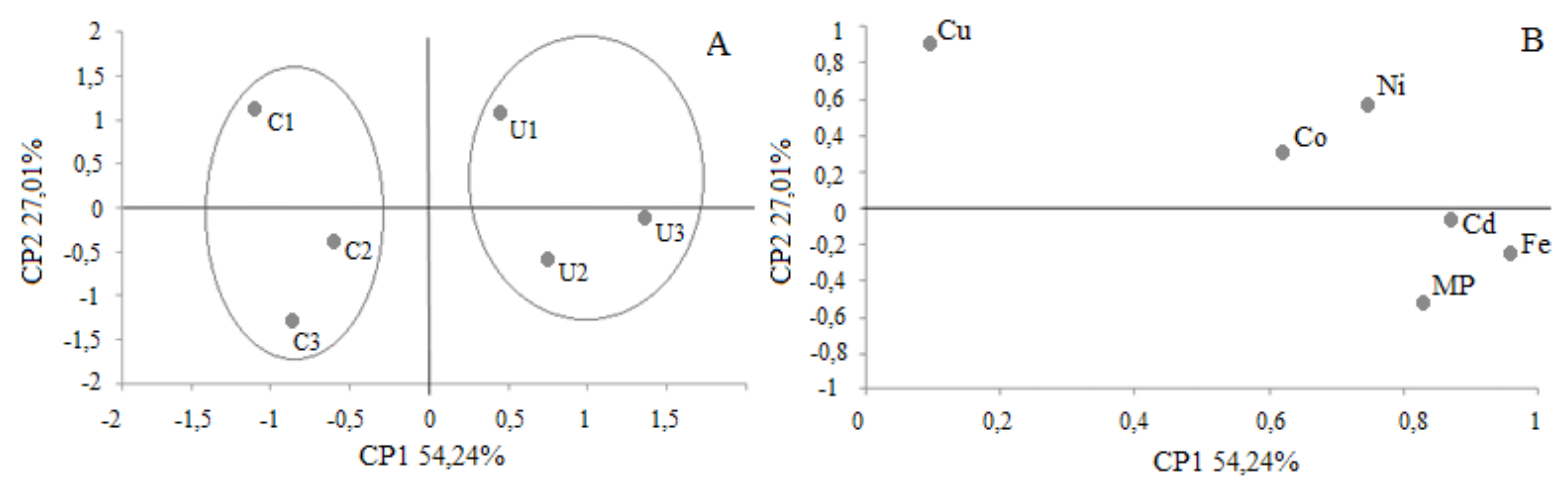

FIGURA 4: Análise de componentes principais (CP1 x CP2): gráfico de escores (A) e loadings (B). $\mathrm{C} 1$ = indivíduo de Terminalia catappa situado no campus, $\mathrm{C} 2$ = indivíduo de Caesalpinia peltophoroides situado no campus, C3 = indivíduo de Bauhinia variegata situado no campus, $\mathrm{U} 1$ = indivíduo de Terminalia catappa situado na avenida, U2 = indivíduo de Caesalpinia peltophoroides situado na avenida, U3 = indivíduo de Bauhinia variegata situado na avenida

FIGURE 4: Principal component analysis: scores plot (A) and loadings plot (B). C1 = indivíduo de Terminalia catappa situado no campus, $\mathrm{C} 2$ = indivíduo de Caesalpinia peltophoroides situado no campus, $\mathrm{C} 3$ = indivíduo de Bauhinia variegata situado no campus, U1 = indivíduo de Terminalia catappa situado na avenida, U2 = indivíduo de Caesalpinia peltophoroides situado na avenida, U3 = indivíduo de Bauhinia variegata situado na avenida

valor superior e positivo quando analisado o eixo da $\mathrm{CP} 2$, instigando assim uma análise mais detalhada desta variável.

\section{Análise de Agrupamento com a Variável Cobre}

De acordo com a Figura 5, pode-se observar a formação de três grupos, sendo agora determinados pela espécie vegetal.

Com base na análise de agrupamento é possível ainda a observação de que as espécies 2 (Caesalpinia peltophoroides) e 3 (Bauhinia variegata) possuem uma maior similaridade quanto ao comportamento das variáveis em relação à espécie 1 (Terminalia catappa). Tal fato pode ser explicado devido às espécies 2 e 3 pertencerem à família Fabaceae. Gilbert (1950) evidenciou que a atividade metabólica do cobre comporta de modo inversamente proporcional à concentração de nitrogênio $(\mathrm{N})$. $\mathrm{O}$ autor destaca que a existência de uma alta concentração de $\mathrm{N}$ na planta impede a translocação do $\mathrm{Cu}$ nas folhas. Mattos Júnior et al. (2010) também demostraram que o suprimento de $\mathrm{Cu}$ em citros é deficiente quando ocorre excesso da adubação nitrogenada. Logo, pela capacidade de espécies florestais pertencentes à família Fabaceae de assimilar N, devido à relação simbiótica com fixadores biológicos (BARBERI et al., 1998), o Cu originado do material particulado é impedido por complexos proteicos/nitrogenados de se translocar na planta, permanecendo acumulado no tecido superficial da folha. Já para a espécie 1 (família Combretaceae), por possuir naturalmente um menor teor de $\mathrm{N}$, permite uma translocação mais eficiente do $\mathrm{Cu}$ para outras partes do vegetal.

\section{Análise de Correlação}

Na Tabela 5 observa-se uma correlação significativa entre o MP e o Fe, mostrando que estes se comportaram de modo similar nas amostras, já que o material particulado advindo de qualquer tipo de fuligem de origem veicular possui Fe em sua constituição (SILVA, 2007). Pode-se perceber também uma forte correlação do $\mathrm{Cd}$ com o Fe, estes elementos estão associados, uma vez que este primeiro é a matériaprima para processos de galvanoplastia em peças automotivas (MOORE; RAMAMOORTHY, 1984). O Cd 


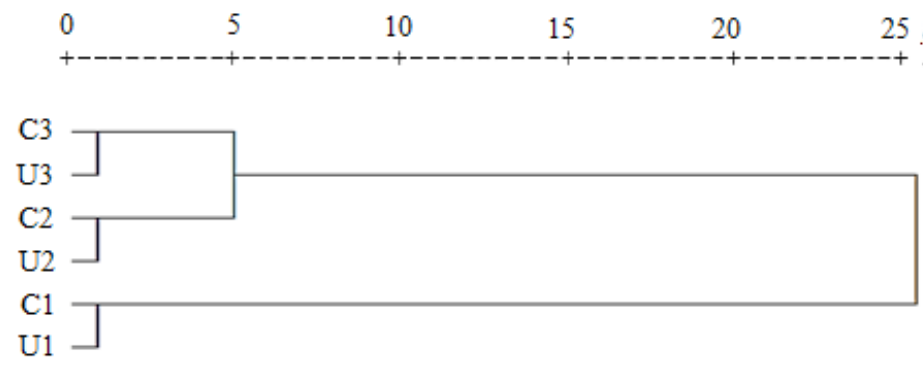

FIGURA 5: Dendrograma de similaridade para o elemento cobre $(\mathrm{Cu})$ para as amostras coletadas no campus da Universidade Estadual do Sudoeste da Bahia e na avenida Olívia Flores. C1 = indivíduo de Terminalia catappa situado no campus, $\mathrm{C} 2$ = indivíduo de Caesalpinia peltophoroides situado no campus, C3 = indivíduo de Bauhinia variegata situado no campus, U1 = indivíduo de Terminalia catappa situado na avenida, U2 = indivíduo de Caesalpinia peltophoroides situado na avenida, U3 = indivíduo de Bauhinia variegata situado na avenida

FIGURE 5: Dendrogram of similarity to the copper element $(\mathrm{Cu})$ for samples collected on the campus of the State University of Southwest Bahia and Avenue Olivia Flores. C1 = indivíduo de Terminalia catappa situado no campus, $\mathrm{C} 2$ = indivíduo de Caesalpinia peltophoroides situado no campus, $\mathrm{C} 3$ = indivíduo de Bauhinia variegata situado no campus, U1 = indivíduo de Terminalia catappa situado na avenida, U2 = indivíduo de Caesalpinia peltophoroides situado na avenida, U3 = indivíduo de Bauhinia variegata situado na avenida

TABELA 5: Matriz de correlação entre as variáveis (MP, Cd, Ni, Fe, Co e Cu) nas amostras de folha, sendo:

* para correlações significativas a 0,05 .

TABLE 5: Matrix of correlation between variables (MP, $\mathrm{Cd}, \mathrm{Ni}, \mathrm{Fe}, \mathrm{Co}$ and $\mathrm{Cu}$ ) in swatches, as follows:

* to significant correlations 0.05 .

\begin{tabular}{ccccccc}
\hline & $\mathrm{MP}$ & $\mathrm{Cd}$ & $\mathrm{Ni}$ & $\mathrm{Fe}$ & $\mathrm{Co}$ & $\mathrm{Cu}$ \\
\hline $\mathrm{MP}$ & 1 & & & & & \\
$\mathrm{Cd}$ & $\mathbf{0 , 7 2 9}$ & 1 & & & & \\
$\mathrm{Ni}$ & 0,318 & 0,483 & 1 & & & \\
$\mathrm{Fe}$ & $\mathbf{0 , 8 9 2 *}$ & $\mathbf{0 , 8 0 7}$ & 0,617 & 1 & & \\
$\mathrm{Co}$ & 0,267 & 0,359 & 0,574 & 0,439 & 1 & \\
$\mathrm{Cu}$ & $-0,333$ & 0,125 & 0,575 & $-0,153$ & 0,133 & 1 \\
\hline
\end{tabular}

também manteve uma forte correlação com o material particulado.

Alterações em correlações negativas também foram observadas, como o $\mathrm{Cu}$ com o $\mathrm{MP}$ e o $\mathrm{Fe}$, todavia, esta não foi uma correlação significativa. No entanto, é possível inferir que o $\mathrm{Cu}$ se comporta de modo independente ao $\mathrm{Fe}$ e a sua diluição no material particulado é dependente da espécie botânica (Tabela 5).

Outras correlações importantes não foram observadas, infere-se assim sob a especificidade de cada elemento químico.

De acordo com Moreira (2010), a floresta urbana pode ser utilizada como instrumento de descontaminação atmosférica, sendo que a interação da floresta com a poluição precisa ser entendida. Devido ao modelo econômico atual e a sua decorrente ação destrutiva para com os recursos naturais (PEREIRA; DIEGUES, 2010), a poluição atmosférica nos centros urbanos tornou-se além de um problema para a saúde pública, uma preocupação com os gastos financeiros oriundos da degradação da vegetação e de construções. Verificou-se neste estudo que biomonitoramento, além de se apresentar como uma técnica 
barata, permitiu aferir sobre áreas impactadas pelo trânsito automotivo, uma vez que, para a maioria dos elementos estudados, a área de maior tráfego de veículos proporcionou uma tendência no maior acúmulo de poluentes. Apontou-se também que as espécies de árvores estudadas, apesar de possuírem capacidade semelhante de retenção dos poluentes atmosféricos, são uma forma de filtrar o ar da cidade e que, de certa forma, interceptaram parte dos componentes químicos que seriam possivelmente inalados pela população, causando provavelmente danos à saúde (CARNEIRO, 2004).

\section{CONCLUSÕES}

O trânsito de veículos influi diretamente na disponibilidade de alguns elementos tóxicos e no aumento de partículas sólidas depositadas sobre folhas de árvores. O potencial de retenção dos poluentes para as três espécies foi semelhante.

O material particulado, o ferro e o cádmio foram as variáveis que melhor explicaram o comportamento das amostras oriundas do meio urbano, estando presentes em maiores teores neste local.

$\mathrm{O}$ elemento químico Fe ocorre em maior quantidade e está presente no material particulado, juntamente com o Cd.

Espécies da família Fabaceae não assimilam o cobre originado da poluição e este tende a ficar com uma maior concentração na superfície foliar.

As folhas de árvores podem ser utilizadas como biomonitores passivos de acumulação e permitem a investigação de impactos ambientais.

As árvores podem ser utilizadas como barreiras naturais que "filtram" o ar citadino.

\section{REFERÊNCIAS}

BARBERI, A. et al. Nodulação em leguminosas florestais em viveiros no sul de Minas Gerais. Cerne, Lavras, v. 4, n. 1, p. 145-153, 1998.

BRASIL. Ministério do Meio Ambiente. $\mathbf{1}^{\circ}$ Inventário nacional de emissões atmosféricas por veículos automotores rodoviários. Relatório final, Brasília: MMA, 2011. 114 p.

BRASIL. Departamento Nacional de Transito. Frota por município 2013. 2013. Disponível em <http:// www.denatran.gov.br/frota2013.htm>. Acesso em: 27 ago. 2014.

BRAUN, S.; APPEL, L. G.; SCMAL, M. A poluição gerada por máquinas de combustão interna movidas à diesel - A questão dos particulados. Estratégias atuais para a redução e controle das emissões e tendências futuras. Química nova, São Paulo, v. 27, n. 3, 2003.

CARNEIRO, C.; REISSMANN, C. B.; MARQUES, R. Comparação de métodos de análise Química de K, $\mathrm{Ca}, \mathrm{Mg}$ e Al em folhas de erva-mate (Ilexpara guariensis St. Hil.). Cerne, Lavras, v. 12, n. 2, jun. 2006.

CARNEIRO, R. M. A. Bioindicadores vegetais de poluição atmosférica: uma contribuição para a saúde da comunidade. 2004. 169 f. Dissertação (Mestrado em Enfermagem em Saúde Pública) - Universidade de São Paulo, Escola de Enfermagem de Ribeirão Preto, Ribeirão Preto, 2004.

CARODE, S.; MATOSO, E.; SANTOS, M. C. A espectrometria atômica e a determinação de elementos metálicos em materiais poliméricos. Química Nova, São Paulo, v. 31, n. 6, p. 1533-1542, 2008.

DUARTE, R. P. S.; PASQUAL, A. Avaliação do cádmio (Cd), chumbo (Pb), níquel (Ni) e Zinco (Zn) em solos, plantas e cabelos humanos. Energia na Agricultura, Botucatu, v. 15, n. 1, 2000.

ESTEVES, F. A. Fundamentos de limonologia. Rio de Janeiro: Interciência, 1988. 575 p.

GILBERT, S. G. A Biochemical basis for copper-nitrogen balance in tung. Plant Physiology, Bethesda, v. 26, n. 2, p. $398-405,1950$.

IBGE. Vitória da Conquista - BA. 2013. Disponível em: <http://cidades.ibge.gov.br/xtras/perfil.php?la $\mathrm{ng}=\&$ codmun $=293330 \&$ search $=$ bahia|vitoria-da conquista|infograficos:-informacoes-completas $>$. Acesso em: 27 ago. 2014.

INOUE M. T.; REISSMANN, C. B. Efeitos da poluição na fotossíntese, dimensões da folha, deposição de particulados e conteúdo de ferro e cobre em alfeneiro (Ligustrum lucidium) da arborização de Curitiba, PR. Revista Floresta, Curitiba, v. 21, n. 12, p. 3-11, 1991.

KLUMPP, A. et al. Um novo conceito de monitoramento e comunicação ambiental: a rede europeia para a 
avaliação da qualidade do ar usando plantas bioindicadoras (EuroBionet). Revista Brasileira de Botânica, São Paulo, v. 24, n. 4, 2001.

MARTINS, A. P. G. Cascas de árvores como biomonitores da poluição atmosférica de origem veicular em parques urbanos da cidade de São Paulo. 2009. 110 f. Tese (Doutorado em Ciências) - Universidade de São Paulo, São Paulo, 2009.

MATTHEWS, P. J. Control of metal application rates from sewage sludge utilization in agriculture. Critical Reviews in Environmental Control, Ohio, v. 14, p. 199-250, 1984.

MATTOS JÚNIOR, D. et al. Nitrogênio e cobre na produção de mudas de citro em diferentes porta-enxertos. Bragantia, Campinas, v. 69, n. 1, p. 135-147, 2010.

MELO, L. C. A.; SILVA, C. A. Influência de métodos de digestão e massa de amostra na recuperação de nutrientes em resíduos orgânicos. Química Nova, São Paulo, v. 31, n. 3, 2008.

MENEZES, E. W.; CATALUÑA. Amostragem do material particulado e fração orgânica volátil das emissões em motor ciclo diesel sem a utilização de túnel de diluição. Química Nova, São Paulo, v. 31, n. 8, 2008.

MOORE, J. W.; RAMAMOORTHY, S. Heavy metals in natural waters. New York: Springer-Verlag, 1984. $328 \mathrm{p}$.

MOREIRA, T. C, L. Interação da vegetação arbórea e poluição atmosférica na cidade de São Paulo. 2010. 79 f. Dissertação (Mestrado em Ciências) - Escola Superior de Agricultura "Luiz de Queiroz", Universidade de São Paulo, Piracicaba, 2010.

PEREIRA, B. E.; DIEGUES, A. C. Conhecimento de populações tradicionais como possibilidade de conservação da natureza: uma reflexão sobre a perspectiva da etnoconservação. Desenvolvimento e Meio Ambiente, Curitiba, n. 22, p. 37-50, 2010.

REGAZZI, A. J. INF 766 - Análise multivariada. Viçosa: Universidade Federal de Viçosa, Centro de Ciências Exatas e Tecnológicas. Departamento de Informática, 2001. 166 p.

RIBANI, M. et al. Validação em métodos cromatográficos e eletroforéticos. Química Nova, São Paulo, v. 27, n. $5,2004$.

SATARUG, S. et al. Cadmium, environmental exposure, and health outcomes. Environmental Health Perspectives, North Columbia, v. 118, n. 2, p. 182-190, feb. 2010.

SILVA, M. F. Emissão de metais por veículos automotores e seus efeitos à saúde. 2007. 156 f. Dissertação (Mestrado em Saúde Pública) - Faculdade de Saúde Pública, Universidade de São Paulo, São Paulo, 2007. SILVA, M. F. Avaliação da qualidade do ar utilizando espécies arbóreas na cidade de Patos-PB. 2011. 63 f. Dissertação (Mestrado em Ciências Florestais) - Universidade Federal de Campina Grande, Patos, 2011.

SKOOG, D. A. et al. Fundamentos de química analítica. 8. ed. São Paulo: [s. n.], 2007.

SOARES FILHO, A. O. Estudo fitossociológico de duas florestas em região ecotonal no planalto de Vitória da Conquista. 2000. 154 f. Dissertação (Mestrado em Ecologia) - Universidade de São Paulo, São Paulo, 2000.

VACCARO, S. et al. Comparação entre três diferentes métodos de análise de tecido vegetal. Boletim de Pesquisa Florestal, Colombo, n. 48, jun. 2004. 